\title{
Antena Planar em F-Invertido com Fenda em L-Deitado Otimizada por Algoritmo Genético
}

\author{
Marcelo N. Kawakatsu, Victor Dmitriev e Dilermando R. de Melo
}

\begin{abstract}
Resumo-Este artigo apresenta uma antena planar em F-invertido (PIFA) modificada, com dimensões geométricas reduzidas e largura de banda (BW) de impedância aumentada. A modificação da PIFA consiste na construção de fendas na placa radiadora (patch). Para análise numérica da antena foi utilizado o método dos momentos (MoM) e para otimização dos seus parâmetros geométricos foi utilizado o algoritmo genético (AG). Como resultados, a altura da PIFA foi reduzida em $20 \%$ e a BW de impedância aumentada em $8 \%$ em relação a PIFA original. Para validação dos resultados utilizou-se o software IE3D da Zeland.
\end{abstract}

Palavras-Chave-Antena Planar em F-Invertido, Método dos Momentos, Largura de Banda de Impedância, Algoritmo Genético.

\begin{abstract}
This paper presents a modified planar inverted-F antenna (PIFA) with reduced geometric dimensions and broaden impedance bandwidth (BW). The PIFA's modification consists of slotting the radiating plate (patch). The antenna was numerically analyzed using the method of moments (MoM) and its geometric parameterizes was optimized using genetic algorithm (GA). As results, the height of the antenna was reduced in $20 \%$ and the impedance $\mathrm{BW}$ increased in $10 \%$. For validating the results, it was used the IE3D commercial software, by Zeland.
\end{abstract}

Keywords-Planar Inverted-F Antenna, Method of Moments, Impedance Bandwidth, Genetic Algorithm.

\section{INTRODUÇÃO}

Em aplicações de dispositivos de comunicação móvel, existe uma grande demanda por antenas

\footnotetext{
Marcelo N. Kawakatsu, Victor Dmitriev e Dilermando R. de Melo, Faculdade de Engenharia Elétrica, Universidade Federal do Pará (UFPA), Belém-PA, Brasil, E-mails: mnkawakatsu@yahoo.com.br, victor@ufpa.br, dilermando@ ufpa.br. Este trabalho foi parcialmente financiado pelo CNPq.
}

miniaturizadas, devido ao pequeno espaço disponível para alocação da antena nesses aparelhos. Nestas aplicações, as PIFAs estão sendo amplamente empregadas devido as suas vantagens construtivas, como perfil baixo, custo reduzido e facilidade de fabricação. Entretanto, as PIFAs convencionais possuem como desvantagem operacional uma largura de banda estreita.

Da teoria de antenas, sabe-se que a BW das antenas planares é prejudicada com a redução da sua altura [1], logo, existe um compromisso entre altura e BW. Neste trabalho foi feita a redução da altura da PIFA e ainda se conseguiu aumentar a BW. Para alcançar este objetivo, foi utilizada uma técnica que consiste na construção de fendas no patch da PIFA, permitindo melhorar o casamento de impedância entre a antena e a estrutura de alimentação (cabo coaxial de 50 ohms).

A otimização da PIFA considerada neste trabalho foi realizada em duas etapas: primeiramente, foi feita a otimização dos parâmetros geométricos para obter a maior BW, sem alterar a altura da antena, em seguida, foi feita uma redução gradual da altura, procurando manter a BW.

\section{GEOMETRIA DA ANTENA}

A geometria da PIFA modificada está mostrada na Fig. 1. Esta antena é constituída por um patch, com dimensões $\mathrm{W}_{\mathrm{p}} \times \mathrm{L}_{\mathrm{p}}$, separado pela altura $\mathrm{h}$ do plano de terra, com dimensões $\mathrm{W}_{\mathrm{g}} \times \mathrm{L}_{\mathrm{g}}$, mas conectado a ele através de uma placa de curtocircuito. No patch é produzida uma fenda com formato de L- deitado, com dimensões e posição definidos por $\mathrm{D}, \mathrm{w}, \mathrm{d}_{1} \mathrm{e} \mathrm{d}_{2}$. $\mathrm{O}$ ponto de alimentação desta antena é determinado por $\mathrm{d}_{3}$. 


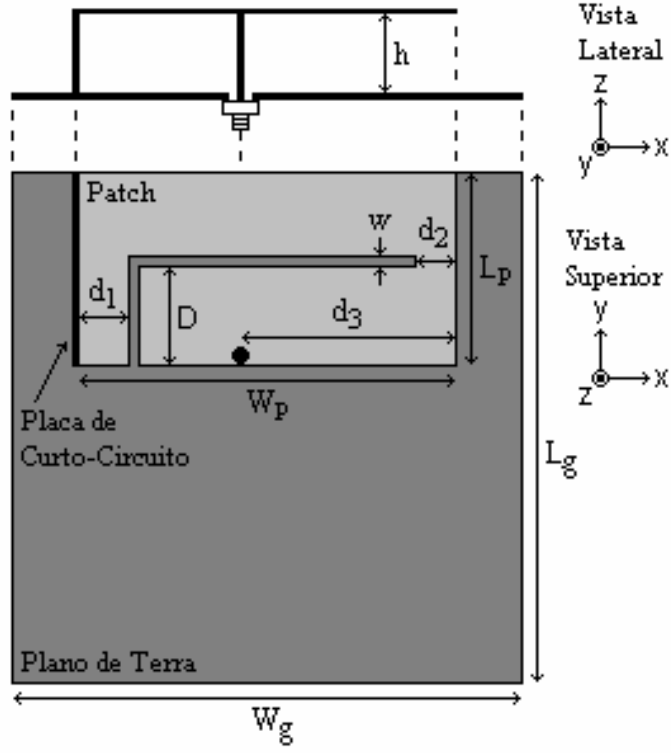

Fig. 1. Esquema da geometria da antena

A configuração geométrica da PIFA originalmente proposta por Chair [2] é definida pelos valores dos parâmetros geométricos mostrados na Tabela I.

TABELA I

PARÂMETROS GEOMÉTRICOS DA PIFA ORIGINAL EM MILÍMETROS

\begin{tabular}{|l|l|l|l|l|l|l|l|l|l|}
\hline $\mathbf{W}_{\mathbf{g}}$ & $\mathbf{L}_{\mathbf{g}}$ & $\mathbf{W}_{\mathbf{p}}$ & $\mathbf{L}_{\mathbf{p}}$ & $\mathbf{h}$ & $\mathbf{D}$ & $\mathbf{W}$ & $\mathbf{d}_{\mathbf{1}}$ & $\mathbf{d}_{\mathbf{2}}$ & $\mathbf{d}_{\mathbf{3}}$ \\
\hline 121 & 127 & 30 & 15 & 10 & 5 & 2 & 2 & 2 & 12 \\
\hline
\end{tabular}

\section{MÉTODOS DE ANÁLISE E OTIMIZAÇÃO}

Para análise numérica da PIFA foi utilizado o MoM baseado nas funções de base Rao-WiltonGlisson (RWG) [3] e [4], implementado no ambiente de programação do MatLab. Este método consiste na resolução das equações de potenciais retardados numericamente através da expansão do integrando (função densidade de corrente nas superfícies metálicas da antena) em uma série finita de termos da função de base. Para aplicação deste método a superfície da antena deve ser discretizada em células unitárias de pequena área, como mostrado na Fig. 2.

Após determinada a distribuição de corrente, é possível determinar os parâmetros de desempenho da antena, como largura de banda de impedância, diagrama de radiação, etc.

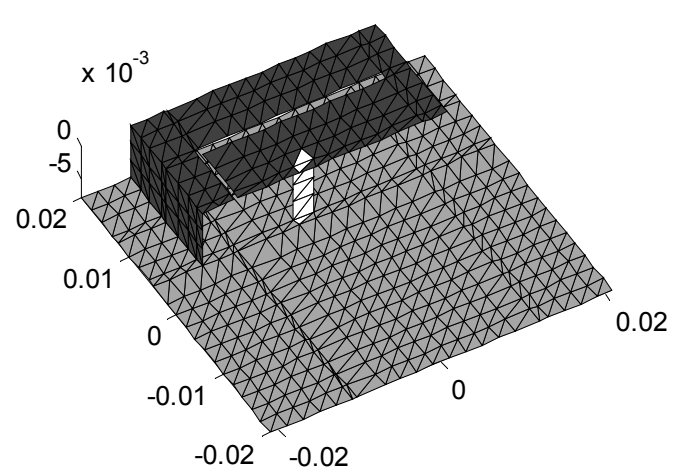

Fig.2. Discretização da superfície da antena para aplicação do MoM

Para otimização da geometria da antena, utilizou-se o AG [5], considerando como função objetivo a maximização da BW. No AG implementado foram considerados as características de elitismo, ponto de cruzamento variável e probabilidade de mutação de $5 \%$. Os parâmetros de otimização considerados foram w, $\mathrm{d}_{1}$, e $\mathrm{d}_{2}$, relacionados com a posição e as dimensões da fenda, e $\mathrm{d}_{3}$, relacionado com a posição do ponto de alimentação. Os demais parâmetros, $\mathrm{W}_{\mathrm{p}}, \mathrm{L}_{\mathrm{p}}, \mathrm{W}_{\mathrm{g}}, \mathrm{L}_{\mathrm{g}}$ e $\mathrm{D}$, foram mantidos fixos com os valores mostrados na Tabela II.

TABELA II

PARÂMETROS MANTIDOS FIXOS. VALORES EM MILÍMETROS

\begin{tabular}{|l|l|l|l|l|}
\hline $\mathbf{W}_{\mathbf{g}}$ & $\mathbf{L}_{\mathbf{g}}$ & $\mathbf{W}_{\mathbf{p}}$ & $\mathbf{L}_{\mathbf{p}}$ & $\mathbf{D}$ \\
\hline 40 & 40 & 30 & 15 & 5 \\
\hline
\end{tabular}

As dimensões do plano de terra, $\mathrm{W}_{\mathrm{g}} \times \mathrm{L}_{\mathrm{g}}$, foram escolhidas tomando como referência as dimensões dos aparelhos de comunicação móveis (celular, por exemplo). As dimensões do patch, $\mathrm{W}_{\mathrm{p}} \times \mathrm{L}_{\mathrm{p}}$, foram mantidas as mesmas da configuração original.

Na primeira etapa, buscou-se obter a maior BW para altura $\mathrm{h}$ de $10 \mathrm{~mm}$, igual à da configuração original. Posteriormente, foram realizadas várias simulações com redução gradual da altura $\mathrm{h}$ de 10 para $6 \mathrm{~mm}$, procurando obter a maior BW para cada valor de $h$, através da otimizando dos parâmetros considerados.

\section{RESULTADOS}

Inicialmente, serão mostrados os resultados de simulação da configuração original mostrada na Tabela I. A impedância de entrada e o coeficiente de reflexão em função da freqüência são mostrados nas Fig. 3 e 4, respectivamente. 


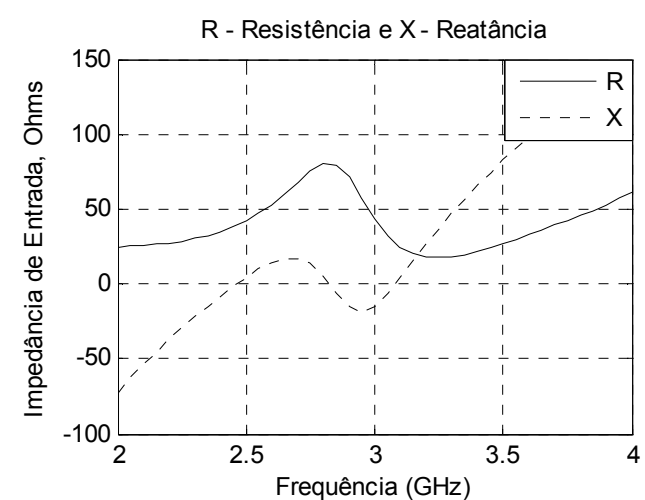

Fig. 3. Impedância de entrada da PIFA original

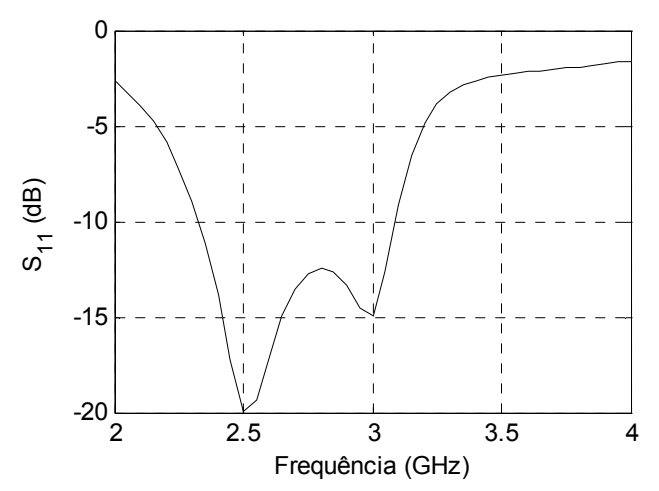

Fig. 4. Coeficiente de reflexão da PIFA original

A BW de impedância da PIFA para esta configuração é de $28,5 \%$, centrada em torno de $2,7 \mathrm{GHz}$, considerando o critério de $-10 \mathrm{~dB}$ para o coeficiente de reflexão $\left(\mathrm{S}_{11}\right)$.

$\mathrm{Na}$ Fig. 5 são mostrados os diagramas de radiação nos planos principais $x-z$ (a) e $y-z$ (b) em $2,7 \mathrm{GHz}$.
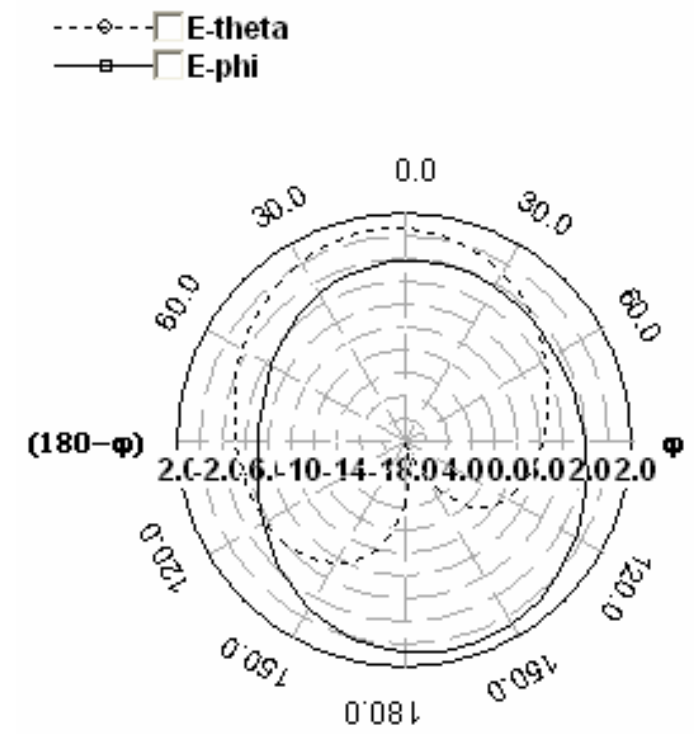

Fig. 5 (a). Diagrama de radiação em 2,7 GHz no plano x-z
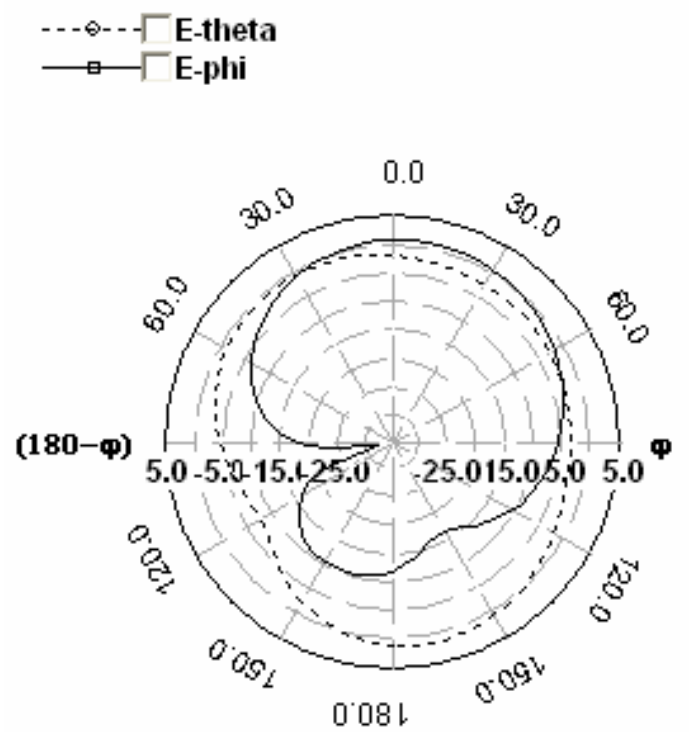

Fig. 5 (b). Diagrama de radiação em 2,7 GHz no plano y-z

A Tabela III mostra a BW de algumas simulações para diferentes valores de $\mathrm{h}$, após a otimização dos parâmetros geométricos $\mathrm{w}, \mathrm{d}_{1}, \mathrm{~d}_{2} \mathrm{e}$ $\mathrm{d}_{3}$ por $\mathrm{AG}$.

TABELA III

LARGURAS DE BANDA OTIMIZADAS PARA DIFERENTES VALORES DE $\mathbf{h}$

\begin{tabular}{|l|l|l|l|l|l|}
\hline h (mm) & 10 & 9 & 8 & 7 & 6 \\
\hline BW (\%) & 36,82 & 33,32 & 30,36 & 23,45 & 14,50 \\
\hline
\end{tabular}

Como pode ser verificado na Tabela III, para altura de $10 \mathrm{~mm}$, igual a da PIFA original, foi possível obter uma BW de 36,82\% (em torno de $30 \%$ maior que a da configuração inicial).

Com a diminuição da altura $\mathrm{h}$, a BW foi reduzida mesmo com a otimização dos parâmetros considerados, como já era esperado. Neste trabalho optou-se pela altura de $8 \mathrm{~mm}$, obtendo-se, dessa forma, uma PIFA com altura relativamente baixa, mas sem comprometer significativamente a BW.

Os valores dos parâmetros otimizados para altura de $8 \mathrm{~mm}$ são mostrados na Tabela IV.

TABELA IV

CONFIGURAÇÃO DA PIFA OTIMIZADA PARA ALTURA DE 8 MILÍMETROS. VALORES EM MILÍMETROS

\begin{tabular}{|l|l|l|l|l|l|l|l|l|l|}
\hline $\mathbf{W}_{\mathbf{g}}$ & $\mathbf{L}_{\mathbf{g}}$ & $\mathbf{W}_{\mathbf{p}}$ & $\mathbf{L}_{\mathbf{p}}$ & $\mathbf{h}$ & $\mathbf{D}$ & $\mathbf{W}$ & $\mathbf{d}_{\mathbf{1}}$ & $\mathbf{d}_{\mathbf{2}}$ & $\mathbf{d}_{\mathbf{3}}$ \\
\hline 40 & 40 & 30 & 15 & 8 & 5 & 0,5 & 4 & 2 & 17 \\
\hline
\end{tabular}

Para esta configuração, a impedância de entrada e o coeficiente de reflexão simulados são mostrados nas Fig. 6 e 7, respectivamente. 


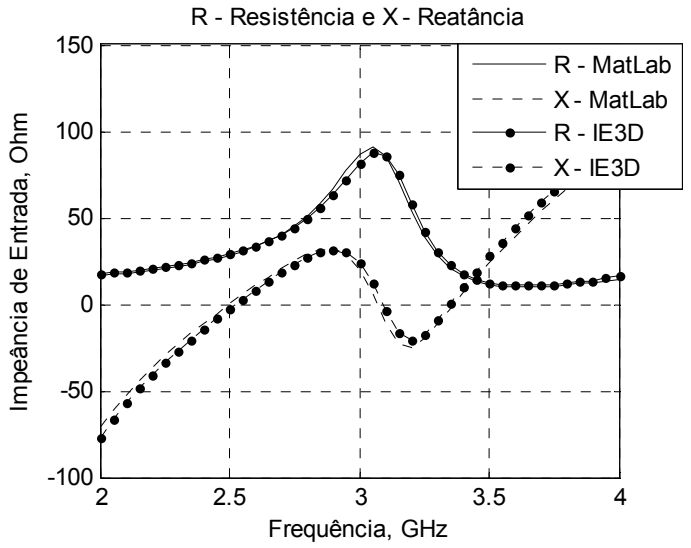

Fig. 6. Impedância de entrada da PIFA otimizada

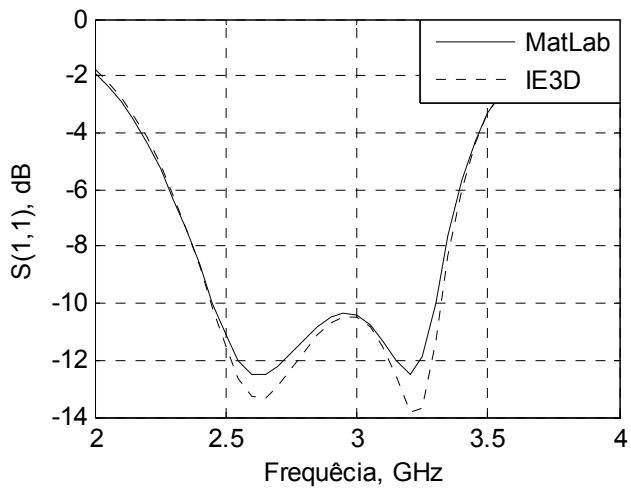

Fig. 7. Coeficiente de reflexão da PIFA otimizada

Utilizando o critério de $-10 \mathrm{~dB}$ para o $\mathrm{S}_{11}$, a PIFA apresentou uma largura de banda de impedância de 30,36 \% centrada em torno de 2,88 GHz.

Os diagramas de radiação obtidos para altura de $8 \mathrm{~mm}$ permaneceram praticamente inalterados em relação os da PIFA original mostrados nas Fig. 5 (a) e (b).

\section{CONCLUSÕES}

Como resultado da primeira etapa, na qual se buscava a maior BW considerando a altura original da PIFA, $10 \mathrm{~mm}$, a otimização por GA dos parâmetros geométricos considerados (dimensões da fenda e posição do ponto de alimentação) permitiu aumentar a BW em torno de $30 \%$ em relação à configuração original. Além disso, este resultado foi obtido considerando um plano de terra de dimensões 40 x $40 \mathrm{~mm}^{2}$, enquanto a configuração original possui um plano de terra de dimensões $121 \times 127 \mathrm{~mm}^{2}$.

$\mathrm{Na}$ segunda etapa, na qual se teve como objetivo a redução da altura da PIFA (miniaturização), mantendo a BW, foi possível reduzir em $20 \%$ esta dimensão (de 10 para $8 \mathrm{~mm}$ ). Mesmo com esta redução, a PIFA otimizada apresentou uma BW em torno de $8 \%$ maior que a da configuração original.

\section{REFERÊNCIAS}

[1] C. Zhi Ning e C. Michael Y. W., "Broadband Planar Antennas", Wiley: UK, 2006.

[2] R. Chair, C. L. Mak, K. M. Luk, e A. A. Kishk, "Wideband Half U-Slot Patch Antennas with Shorting Pin and Shorting Wall", IEEE Transactions on antennas and propagation, 2004.

[3] Sergey N. Makarov, "Antenna and EM Modeling with MatLab”, Wiley: New York, 2002.

[4] Sergey Makarov, "MoM Antennas Simulations with Matlab: RWG Basis Function", IEEE Transactions on antennas and propagation, 2001.

[5] H. Randy L., "An Introduction to Genetic Algorithms for Eletromagnetics", IEEE Transactions on antennas and propagation, Vol. 37, No. 2, 1995. 